\title{
Review
}

\section{Ultra-slow relaxation in ice and related substances}

\author{
By Hiroshi SugA *),**) \\ Professor Emeritus, Osaka University \\ (Communicated by Syûzô SEKI, M. J. A.)
}

\begin{abstract}
Of prime interest in numerous studies on water, an important substance to all living systems, may be its physical, chemical, biological characteristics in our internal and external environments. One of the central problems underlying all these researches is a basic structural problem. An important question was why ordinary ice did not obey the third law of thermodynamics. The proton-disordered phase $\mathrm{I}_{\mathrm{h}}$ remains down to the lowest temperature without exhibiting any indication of phase transition. We found that the glass transition is not a characteristic property of liquid but of wide occurrence in condensed systems that failed to maintain thermal equilibrium during continuous cooling. Crystalline substance that exhibited freezing-in process on cooling was designated as "glassy crystals". In fact, ice $\mathrm{I}_{\mathrm{h}}$ exhibited a glass transition at around $110 \mathrm{~K}$ due to slowing down of reorientational motion of the water molecules. A particular kind of impurity was found to accelerate dramatically the motion, and to induce a long-awaited phase transition at $72 \mathrm{~K}$. The transition removed a substantial fraction of the residual entropy of ice. The dopant acted as a kind of catalysis for releasing the immobilized non-equilibrium state to recover thermal equilibrium in the laboratory time. Structure and some properties of the ordered low temperature phase, designated as ice $\mathrm{XI}$, are discussed. The same ordering processes observed in some clathrate hydrates possessing hydrogenbonded networks similar to ice $\mathrm{I}_{\mathrm{h}}$ are described. Interplay between phase transformation and glass transition in relation to important role played by particular dopant is discussed.
\end{abstract}

Key words: Phase transition; glass transition; heat capacity and entropy; ice; clathrate hydrate; dopant.

Introduction. A wide range of substances can be vitrified by cooling their liquids. At temperatures well below the fusion point, the relaxation time for viscous flow becomes so long that the liquid ultimately behaves like a solid. The glassy states of liquid are found to exhibit the glass transition $T_{\mathrm{g}}$ and residual entropy $S_{0}$ as indicative of non-ergodicity of the frozen-in non-equilibrium system. ${ }^{1)}$ The liquid undergoes a heat capacity discontinuity over a narrow temperature interval of $T_{\mathrm{g}}$, where the relaxation time for the structural change in a liquid becomes of the order of $1 \mathrm{ks}$ that corresponds to the time scale for a single heat-capacity determination. Corresponding to the $C_{p}$ drop, the entropy of the liquid

\footnotetext{
*) Home address; Minoo 4-17-39, Osaka 562-0001, Japan. E-mail; qxprp464@ybb.ne.jp

**) Recipient of the Japan Academy Prize in 1995.
}

bends at $T_{\mathrm{g}}$ and results in residual entropy $S_{0}$ that is a measure of the extent of frozen-in disorder. These quantities are highly time-dependent. If the liquid were cooled with an infinitely slow rate, the liquid follows the extrapolated curve of entropy of the undercooled liquid and finally intersects that of the corresponding crystal. Since any disordered system with less entropy than that of the crystal cannot be considered, the entropy of the equilibrium liquid must bend at this temperature in order to avoid the entropy crisis. The temperature is known as the Kauzmann temperature $T_{\mathrm{K}}$. Thus the seemingly second-order character of the glass transitions in real liquids is believed to be a kinetic manifestation of the underlying second-order phase transition in the "equilibrium" liquid.

Essentially the same phenomena were found to occur also in some crystalline solids that have orienta- 
tional degrees of freedom. ${ }^{2)}$ Frozen-in state of orientational disorder of the constituent molecules in crystal can be realized by rapid cooling of high temperature disordered phase for avoiding the transformation into an ordered low temperature phase. This was typically observed in cyclohexanol that crystallizes into an fcc phase. The glass transition was observed at $150 \mathrm{~K}$ with a large drop of heat capacity in the undercooled fcc phase. Although crystals and glassy liquids are two extremes in our conception of structural regularity in the solid state, the frozen-in orientationally disordered state of the undercooled cyclohexanol crystal was designated as the glassy crystal. ${ }^{3)}$ Another category of frozen-in disordered state can be found in stable crystalline phase, as in the cases of $\mathrm{CO}$ and $\mathrm{H}_{2} \mathrm{O}$ crystals. Discovery of these glassy crystals required a renewal of the concept of glass transition, which should occur widely in any disordered systems whenever the relaxation time for some degrees of freedom of the system crosses the experimental time. It is not unusual during continuous cooling of any disordered system for some aspects of its to fail to maintain thermal equilibrium.

Water is the most abundant liquid compound found on the surface of our globe. ${ }^{5)}$ The solid form of water, hexagonal ice $I_{h}$ at atmospheric pressure, exists mainly in the polar region and partly in the Greenland and various glaciers. The term "crystal" is derived from the Greek "Krustallos" that meant ice. Ice has long been accepted as a typical crystalline substance surrounding us. Meanwhile, ice was found to deviate from the third law of thermodynamics, which is obeyed by crystals in most cases. The exception of ice from the law attracted many scientists, but nobody could find out any heat-capacity anomaly that can be ascribable to an ordering transition. The residual entropy of ice $\mathrm{I}_{\mathrm{h}}, S_{0}=$ $3.4 \mathrm{~J} \mathrm{~K}^{-1} \mathrm{~mol}^{-1}$, was interpreted in terms of the halfhydrogen statistical model based on the ice rules. ${ }^{6)}$ The rules state that (1) $\mathrm{H}_{2} \mathrm{O}$ molecules in ice are intact, (2) each molecule is hydrogen bonded with four neighboring molecules, and (3) each hydrogen bond contains only one proton. Obviously this kind of statistical model cannot be an equilibrium structure at $0 \mathrm{~K}$. Why ice $\mathrm{I}_{\mathrm{h}}$ does not realize an ordered state? The problem induced heated discussions. Some scientists proposed from a computer simulation that the proton-disordered phase $I_{h}$ is energetically favored over any proton-ordered structures. Such a bold proposal will shake the firm basis of the third law of thermodynamics.

Dynamic situation of water reorientational motion can be inferred from the dielectric measurements. The dipolar molecules can contribute from orientation polarization to the dielectric permittivity at high temperatures under an ac electric field with appropriate frequency. On cooling, the prolongation of the dielectric relaxation time hinders the crystal from responding to the sinusoidal change in external frequency below certain temperature region that depends on the external frequency. Only the contribution from the electronic polarization remains below that region. The dielectric relaxation provides us with knowledge on the dynamics by which a dipolar molecule reorients in the crystal. On the other hand, an adiabatic calorimeter turned out to work as a low-frequency spectrometer to determine the enthalpy relaxation time in slow regime. ${ }^{7}$ Both the calorimetric and dielectric relaxation times turned out to be correlated well each other. The present article describes ultra-slow dynamics of ice and clathrate hydrates, and dopant-induced phase transitions observed in them. A particular kind of impurity doped into the lattice acted on a frozen-in disordered system as catalysis for releasing the immobilized state and recovering the equilibrium state in our observation time. ${ }^{8)}$

Adiabatic calorimeter as a low-frequency spectrometer. The adiabatic calorimeter has proven its usefulness in studies of the relaxation processes in glassy liquids and other frozen-in disordered systems. Exothermic or endothermic temperature changes arising from the enthalpy relaxation can be observed around $T_{\mathrm{g}}$, depending on whether the actual frozen-in enthalpy locates above or below the equilibrium value. In an ordinary operation of the adiabatic calorimeter with a discontinuous heating mode, the measurement of heat capacity is a repetition of the "equilibration" and "energizing" periods. In the former period, the calorimetric temperature is recorded as a function of time. Constant temperature over a period of time is the necessary condition of thermal equilibrium. This temperature is referred to initial temperature $T_{\mathrm{i}}$. A definite amount of electric energy $E$ is supplied to the calorimeter. The next "equilibration" period is followed to determine a new equilibrium temperature $T_{\mathrm{f}}$, which becomes $T_{\mathrm{i}}$ for the subsequent measurement. The average heat capacity $C_{p}$ is determined by $E /\left(T_{\mathrm{f}}-T_{\mathrm{i}}\right)$. The data are manipulated to derive the enthalpy, entropy, and Gibbs energy of the sample. The accuracy of our $C_{p}$ data is determined by measuring the heat capacities of the standard benzoic acid crystal supplied by NBS (now NIST) and to compare them with the values recommended by international community. The accuracy is $\pm 1 \%$ below $20 \mathrm{~K}, \pm 0.5 \%$ between 20 and $50 \mathrm{~K}$, and $\pm 0.3 \%$ above $50 \mathrm{~K}$. Another 
Table I. Standard molar entropy of $\mathrm{SF}_{6}$ (g) at $298.15 \mathrm{~K}$

\begin{tabular}{lc}
\hline$T / \mathrm{K}$ & $\Delta S_{\mathrm{m}}^{\circ} / \mathrm{J} \mathrm{K}^{-1} \mathrm{~mol}^{-1}$ \\
\hline 0 90.8 (integration of $\left.C_{p} / T\right)$ & 75.35 \\
$90.8 \sim 98.2$ (transition) & 22.62 \\
98.2 186.0 (integration of $C_{p} / T$ ) & 44.26 \\
186.0 sublimation $(p=18.6 \mathrm{kPa})$ & 124.8 \\
Ideal gas correction & 0.175 \\
186 298.15 (integration of $\left.C_{p} / T\right)$ & 37.85 \\
Compression to $10^{5} \mathrm{~Pa}$ & -13.98 \\
Calorimetric entropy & $291.2 \pm 0.5$ \\
Statistical entropy & $291.6 \pm 0.4$ \\
\hline
\end{tabular}

method is to compare the calorimetric entropy with the statistical one determined independently. This was done for $\mathrm{SF}_{6}$. The crystalline $\mathrm{SF}_{6}$ exhibited a first-order phase transition at $95.02 \mathrm{~K}$ and fusion at $223.72 \mathrm{~K}$. The enthalpy of sublimation at $186.00 \mathrm{~K}$ was determined calorimetrically to be $(23.22 \pm 0.01) \mathrm{kJ} \mathrm{mol}^{-1}$. Table I lists the molar entropies of $\mathrm{SF}_{6}$ gas in an ideal state calculated in this way. ${ }^{9)}$ The calorimetric value agrees well with the literature datum of the statistical entropy, showing that the low temperature phase of $\mathrm{SF}_{6}$ has an ordered structure.

If the calorimetric temperature during the equilibration period happens to change with time, this means that some irreversible processes are occurring in the sample. This is typically observed in glassy liquid that was brought into frozen-in disordered state as a consequence of the failure of the kinetic process by which the configurational degree of freedom of the liquid is brought into correspondence with the temperature of the remaining vibrational degrees of freedom. The excess configurational enthalpy $H_{\mathrm{c}}$ stored in the glassy liquid tends to relax towards the equilibrium value. This causes spontaneous rise of the calorimetric temperature under the adiabatic condition. The exothermic process continues over an extremely long time at low temperatures, so that the next energizing is started before the equilibrium is established.

The cycle is repeated until the enthalpy of the system crosses apparently the equilibrium value. The subsequent energizing induces the system to deviate from the equilibrium in the opposite direction, thus showing spontaneous temperature falling in the subsequent equilibration periods. Finally the system reaches the equilibrium liquid at which the structural relaxation time becomes short enough to realize the thermal equilibrium in a short period. Thus a series of exothermic followed by endothermic processes can be observed in a

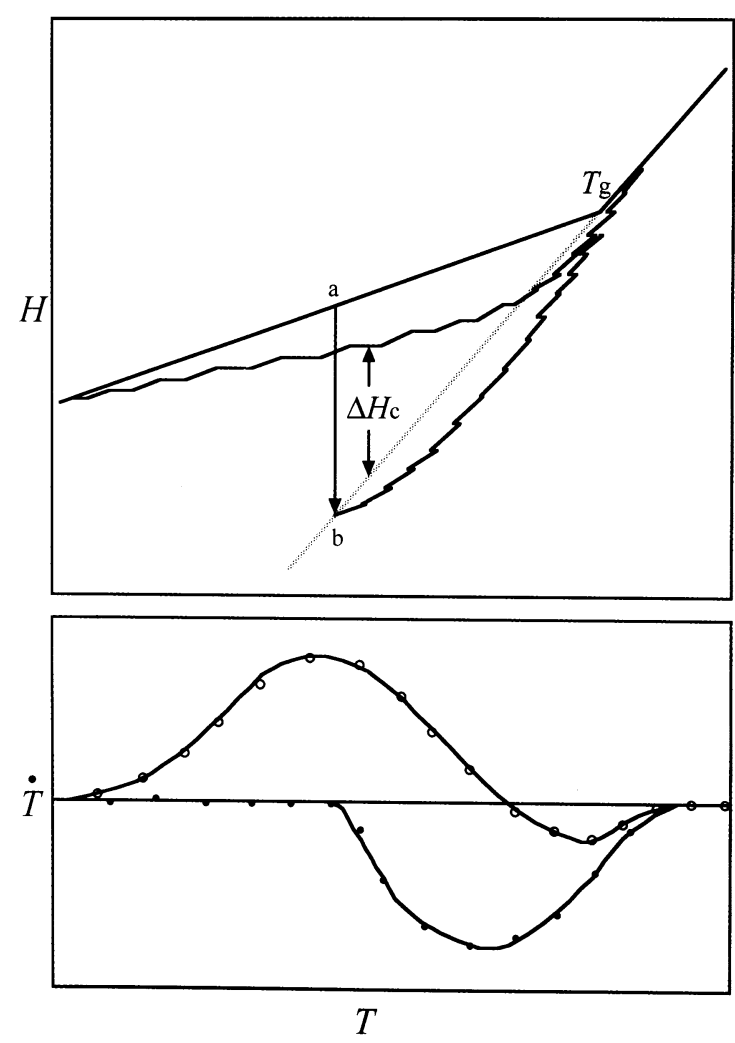

Fig. 1. (Top) Schematic drawing of enthalpy $H$ versus temperature $T$ curve of a substance that undergoes a glass transition $T_{\mathrm{g}}$. Zigzag line indicates thermal path that a glassy sample follows during heat capacity measurements. (Bottom) Initial rate of spontaneous change in the calorimetric temperature observed during equilibration period.

narrow temperature range around $T_{\mathrm{g}}$ of the liquid. The situation is drawn schematically in Fig. 1. It is clear that a well-annealed sample (say, point $\mathrm{b}$ in the figure) exhibits only endothermic temperature changes during every equilibration periods on warming.

The relaxation function for the configurational enthalpy $H_{\mathrm{c}}$ is well described by the KohlrauschWilliams-Watts' (KWW) equation. ${ }^{1)}$

$$
\Delta H_{\mathrm{c}}(t)=\Delta H_{\mathrm{c}}(0) \exp \left[(-t / \tau)^{\beta}\right]
$$

where $\Delta H_{\mathrm{c}}(0)$ and $\Delta H_{\mathrm{c}}(t)$ are the enthalpy to be relaxed at time 0 and $t, \tau$ is the average relaxation time, $\beta$ is the non-exponential parameter representing a distribution of the relaxation times. The remaining part of the enthalpy, called the vibrational enthalpy $H_{\text {vib }}$, responds always quickly to the temperature change. Since the total enthalpy of a sample, $H_{\text {total }}=H_{\text {vib }}+H_{\mathrm{c}}$, can 


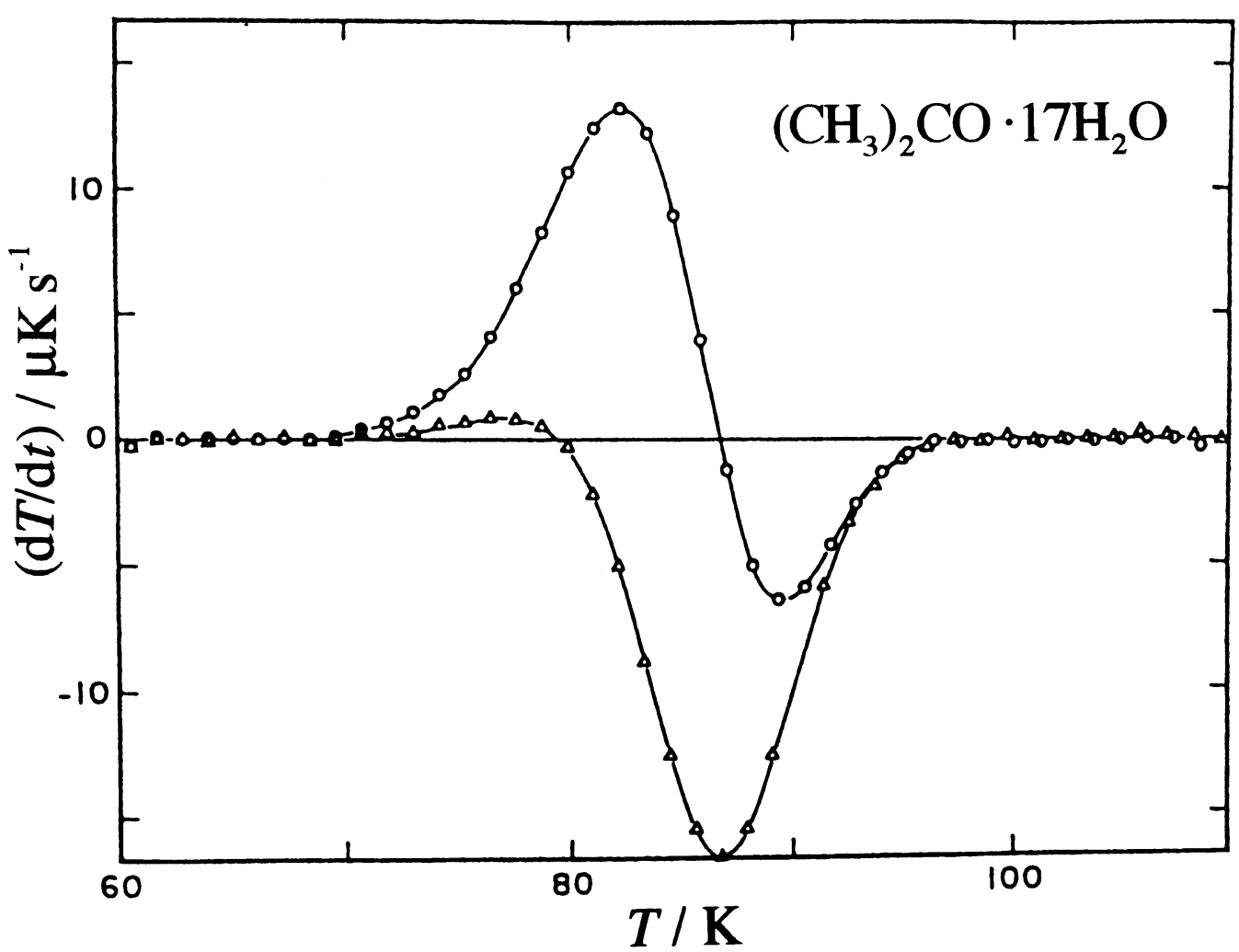

Fig. 2. Spontaneous temperature drift rate observed during equilibration period of acetone clathrate hydrate $\left(\mathrm{CH}_{3}\right)_{2} \mathrm{CO} \cdot 17 \mathrm{H}_{2} \mathrm{O}$. Symbol $\bigcirc$ is for a crystal cooled at $-2.4 \mathrm{~K} \mathrm{~min}^{-1}$ and symbol $\triangle$ for an annealed crystal at $80 \mathrm{~K}$ for $30 \mathrm{~h}$ after the above experiment.

be kept constant under an adiabatic condition, it follows

$$
\mathrm{d} \Delta H_{\mathrm{c}}(t) / \mathrm{d} t=-\left[\mathrm{d} \Delta H_{\mathrm{vib}}(t) / \mathrm{d} T\right][\mathrm{d} T / \mathrm{d} t]=-C_{\text {vib }}[\mathrm{d} T / \mathrm{d} t]
$$

Then the following equation is derived for the calorimetric temperature as a function of time.

$$
T_{\mathrm{vib}}(t)=a+b t+c \exp \left[(-t / \tau)^{\beta}\right]
$$

Here $a, b$ and $c$ are the constants to be determined by experimental conditions. The second term bt represents the temperature drift rate due to residual heat leakage and is of the order of several $\mathrm{mK} \mathrm{h}^{-1}$ in most of the temperature range. The value $C_{\text {vib }}$ can be determined by extrapolating the temperature-time curve to the mid-point of energizing periods so as to erase the relaxation effect. Thus equation [3] enables us to determine experimentally the two kinetic parameters, $\tau$ and $\beta$, governing the relaxation process by analyzing the calori- metric temperature $T$ observed as a function of time $t$. In fact, this method was applied to isocyanocyclohexane ${ }^{10)}$ around its $T_{\mathrm{g}}$, and the following data were successfully determined; $\boldsymbol{\tau}=8.3 \mathrm{ks}$ and $\beta=0.70$ at $125.37 \mathrm{~K}, \boldsymbol{\tau}=$ $4.7 \mathrm{ks}$ and $\beta=0.75$ at $126.82 \mathrm{~K}, \tau=2.5 \mathrm{ks}$ and $\beta=0.81$ at $127.66 \mathrm{~K}$. In this way, the adiabatic calorimeter works as an ultra-low frequency spectrometer for studying slow dynamics in condensed matters. The time domain covered by the method ranges $10^{2}$ to $10^{6} \mathrm{~s}$, depending on the quality of the calorimeter and the patience of the experimenter. Thus the calorimetric method is complementary to dielectric, NMR and other spectroscopic methods that give kinetic parameters in fast regime. Figure 2 represents the actual data of the spontaneous temperature changes arising from enthalpy relaxation observed for acetone clathrate hydrate for a rapidlycooled and a well-annealed sample, respectively. The crystal belongs to one category of the glassy crystals. The thermal behavior around its $T_{\mathrm{g}}(=90 \mathrm{~K})$ is essentially the same as those observed in glassy liquids. Details of the 
experiment are described later.

Hexagonal ice. The apparent beauty of ice single crystal is marred by the disordered nature of protons at the molecular level. Each water molecules, possessing two proton-donating and two proton-accepting ability, can have six orientations in the lattice, in which every molecules are surrounded tetrahedrally by four neighboring molecules through the hydrogen bonds. Since each hydrogen bond can accommodate only one proton at a time (one of the ice rules), the reorientational motion of water molecules must be synchronized as a whole. It is this highly cooperative nature of the coupled motion that prolongs the relaxation time for the motion and hinders the crystal to realize a hypothetical ordering transition at a low temperature. Relaxation process of ice associated with $T_{\mathrm{g}}$ at $110 \mathrm{~K}$ was studied by the calorimetric measurement. ${ }^{11)}$ The enthalpy relaxation time thus determined is as follows: $\tau=3.67 \mathrm{ks}$ at $107.6 \mathrm{~K}, 13.8$ $\mathrm{ks}$ at $102.5 \mathrm{~K}, 49.0 \mathrm{ks}$ at $97.4 \mathrm{~K}, 0.52 \mathrm{Ms}$ at $89.4 \mathrm{~K}$. These calorimetric relaxation data can be correlated well with the literature data of the dielectric relaxation time in an Arrhenius plot, ln $(\tau / \mathrm{s})$ vs $1 / T$. A straight line can connect both the calorimetric and dielectric data plotted over a wide range of relaxation time. This means that the calorimetric and dielectric measurements observed a relaxation process of the same molecular motion with different time windows. The activation energy derived from the line was $21 \mathrm{~kJ} \mathrm{~mol}^{-1}$. If we extrapolate these data to $60 \mathrm{~K}$, a hypothetical transition temperature of ice calculated by Pitzer and Polissar ${ }^{12)}$ based on a molecular field theory, we immediately recognize that achievement of an ordered ice requires about $10^{13} \mathrm{~s}$, a geological time.

A hint how to resolve the proton ordering was given by dielectric studies on doped ices. The water reorientational motion in the lattice is believed to occur through a lattice imperfection designated as the D (doppelt) and L (leer) orientational defects. The rotation of a water molecule due to thermal fluctuation around one of the hydrogen bonds by $(2 / 3) \pi$ radians produces a pair of unusual hydrogen bonds; one contains two protons and the other no proton in the neighboring two hydrogen bonds. This energized state that temporarily breaches the ice rules, returns to the normal state by recombination of both defects through encounter at some places after a step-by-step movement of the defects along the hydrogen bonds. During the travel of both defects, water molecules along the path can change their orientations, thus facilitating the reorientational motion of water molecules. The property of such intrinsic defects was clarified by the dielectric measure-

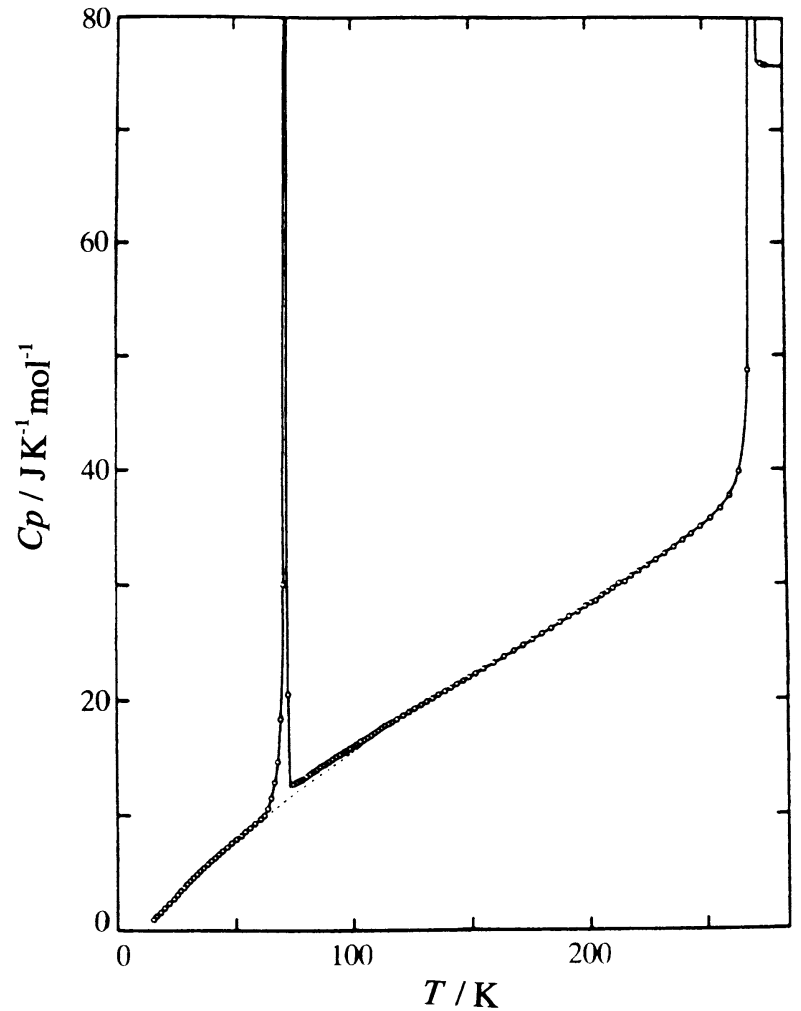

Fig. 3. Heat capacity of ice $\mathrm{I}_{\mathrm{h}}$ doped with $\mathrm{KOH}$ in the mole fraction of $1.8 \times 10^{-4}$

ment of ice doped with particular impurity that creates the orientational defects. ${ }^{13)}$ A special kind of dopant was found to relax the severe constraints imposed on the reorientational motion and shortens dramatically the dielectric relaxation time. After several trying, $\mathrm{KOH}$ was chosen as a dopant. The hydroxide ion can replace the water molecules in the ice lattice and creates an ionized vertex together with an orientational L defect. The negatively charged $\mathrm{OH}^{-}$ion will attract neighboring protons more easily, and is expected to reduce effectively the activation energy for the movement. Contrary to the intrinsic defects, the concentration of the L defects created by artificial doping is independent of temperature. Thus the acceleration effect of the defect on the orientational mobility of water molecules in the doped sample is expected to remain down to the low temperature region.

Three kinds of KOH-doped ice sample with mole fraction $x$ of $1.8 \times 10^{-5}, 1.8 \times 10^{-4}$, and $1.8 \times 10^{-3}$ were prepared. Each sample was annealed at $65 \mathrm{~K}$ where some common exothermic effects ascribable to the 


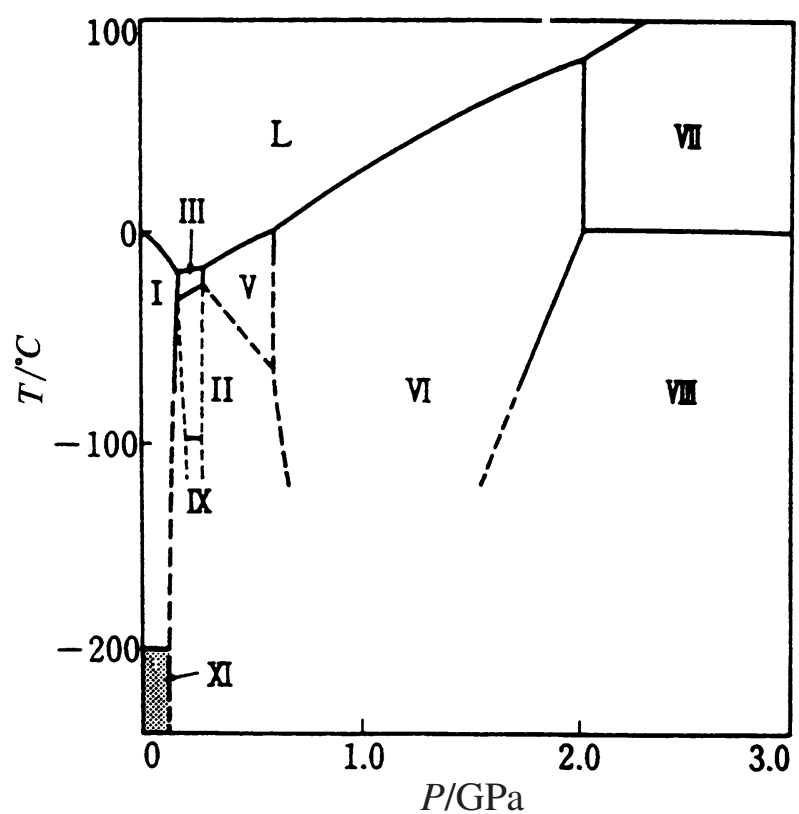

Fig. 4. Phase diagram of ice.

onset of the ordering were found. The annealing was continued for three days until the exothermic process almost ceased. The subsequent measurement exhibited a first-order phase transition at $72 \mathrm{~K}$ which has long been looked for. Typical result for the sample $x=1.8 \times 10^{-4}$ is shown in Fig. $3{ }^{14)}$ The heat capacity associated with the transition has a long tail that continues up to $120 \mathrm{~K}$. It was this high-temperature tail that was observed as a small jump in the heat capacity measurement of pure ice. Thus the freezing out of water motion occurred at the very initial stage of short-range ordering. The transition temperature was independent of the amount of the dopant, but the corresponding entropy change $\Delta_{\text {trs }} S$ increased with the amount of $\mathrm{KOH}$. The maximum entropy of transition $2.33 \mathrm{~J} \mathrm{~K}^{-1} \mathrm{~mol}^{-1}$ was obtained for the sample doped with $1.8 \times 10^{-3}$ mole fraction (maximum solubility) of $\mathrm{KOH}$ annealed for $256 \mathrm{~h}$. Thus the transition removed a substantial fraction of the residual entropy. It is conjectured that the limited solubility of $\mathrm{KOH}$ into the ice lattice prevents the crystal from completing the transformation into the ordered ice.

The new modification of proton-ordered ice was designated as ice XI, following the discovery of other modifications. Phase boundary between $\mathrm{I}_{\mathrm{h}}$ and XI was determined by a high-pressure calorimeter workable to $250 \mathrm{MPa}$ using $\mathrm{He}$ gas as the pressure-transmitting medium. ${ }^{15)}$ Pressure dependence of the transition tem-

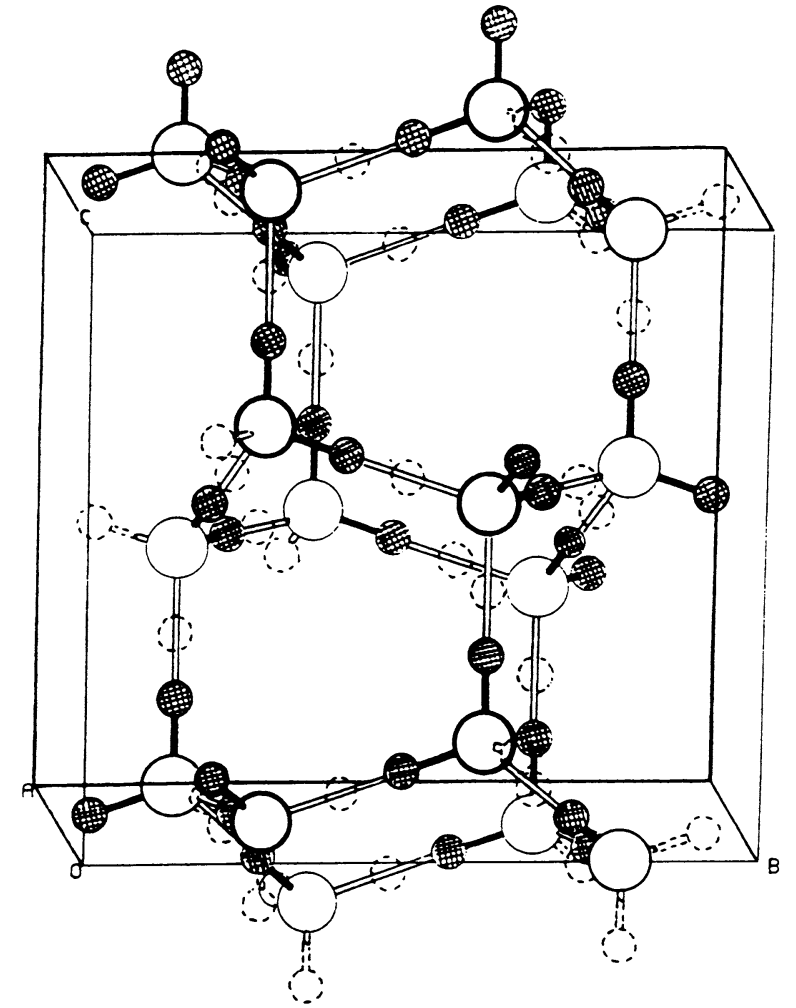

Fig. 5. Structure model of ice XI. Oxygen (large circles) remains in the same spatial arrangement. Deuterons (hatched small circles) have unit site occupancy in the Cmc $2_{1}$ space group. Ice $I_{h}$ is obtained when each deuterium has site occupancy of 0.5. The occupancies of deuteriums lie between 0.5 and 1 in a partially ordered state.

perature turned out to be $\mathrm{d} T_{\mathrm{trs}} / \mathrm{d} p=(0.015 \pm 0.001) \mathrm{K}$ $\mathrm{MPa}^{-1}$. The result combined with the ClausiusClapeyron's equation yields the value $\Delta_{\text {trs }} V$ to be $(0.051 \pm$ $0.003 \mathrm{~cm}^{3} \mathrm{~mol}^{-1}$, which corresponds to $0.26 \%$ of the total volume. The small value in the slope $\mathrm{d} T_{\text {trs }} / \mathrm{d} p$ is consistent with the corresponding ordering transitions in ice III and VII. Both modifications have disordered structures with respect to protons and undergo the corresponding transition at 173 and $278 \mathrm{~K}$ to form ice IX and VIII, respectively. Compared to these temperatures, the ordering temperature of $\mathrm{I}_{\mathrm{h}}, 72 \mathrm{~K}$, is too low to realize the possible ordering in the laboratory time in pure state. Phase diagram of $\mathrm{H}_{2} \mathrm{O}$ including water and ice is shown in Fig. 4.

A neutron diffraction experiment was carried out on KOD-doped $\mathrm{D}_{2} \mathrm{O}$ ice $\left(x=1.8 \times 10^{-3}\right)$ that undergoes the ordering transition at $76 \mathrm{~K}^{16)}$ The positive deuteron isotope effect of $4 \mathrm{~K}$ on the $T_{\text {trs }}$ is generally observed in 
hydrogen-bonded crystals with $\mathrm{O} \cdots \mathrm{O}$ distances of the order of $275 \mathrm{pm}$. The space group for the ice XI was $\mathrm{Cmc2}_{1}(z=4)$. The structure model for the orthorhombic ice XI and its relation to ice $I_{h}$ are illustrated in Fig. 5 . The structure is polar along the $c$ axis of the original hexagonal unit cell. The marked broadening of the extra peak associated with the proton ordering and the diffuse wings underlying several other peaks imply a small size of domain and possibly some strain of the ordered region that coexist with disordered $\mathrm{I}_{\mathrm{h}}$. Analysis of the line profiles shows that the average size of the ordered domains is about $40 \mathrm{~nm}$ in extent. Most probably, one dopant entity acted as a catalytic particle in the ice lattice by inducing the ordering transition in $10^{4}$ of the surrounding water molecules. ${ }^{17)}$ Very little is known about the role of $\mathrm{K}^{+}$ion in the ice lattice. Potassium ion has exhibited the largest acceleration effect among alkali ions examined. It has been calculated that a potassium cation finds a low energy site at the center of the hexagonal ring formed by six water molecules in a clathrate-like structure. If such a site is available to the potassium ion in the ice lattice, the hydrogen-bonded network will be greatly disturbed around the cation. This will produce a large number of defects in the lattice which obviate the ice rules locally. This will in turn promote the ordering of neighboring water molecules that has been suppressed for kinetic reason. Both of the cations and anions must contribute to the ordering process in ice crystal in a conjugate way.

A later neutron diffraction experiment gave the same space group. ${ }^{18)}$ These authors attempted to interpret their diffraction data in terms of the other 17 orthorhombic structures tabulated by Howe. ${ }^{19)}$ None of these was compatible with the observed profiles, and the Cmc2 ${ }_{1}$ structure (designated as C-ice) provided much the best fit to their diffraction data. On the other hand, Davidson and Morokuma ${ }^{20)}$ proposed an antiferroelectrically ordered structure with a Pna $2_{1}$ space group $(z=$ 8 ) as the most stable ordered form of ice (P-ice). Their consideration is based on the stable conformation of a water dimer. They built up the dimer step by step so as to minimize the interaction energy and concluded that the P-ice was more stable by $8 \mathrm{~kJ} \mathrm{~mol}^{-1}$ than the ferroelectrically ordered C-ice. Pisani et al. carried out a quantum-mechanical computation on the two possible structures. ${ }^{21)}$ The equilibrium geometry and relative stability of the structure were examined by performing periodic Hartree-Fock calculations. The main conclusion is that the energy difference between the two structures is within the estimated computational uncertainty and they may be considered to be iso-energetic. Interestingly the role of $\mathrm{KOH}$ is such that the dopant not only enhances the orientational mobility of water molecules but also significantly stabilizes the C-ice. This stabilization will support the assumption that micro-crystals or small-sized domains of ice XI are formed around the individual dopant entities. Jackson and Whitworth ${ }^{22)}$ observed a peak in thermostimulated depolarization current at $72 \mathrm{~K}$ in a single crystal of $\mathrm{KOH}$-doped ice. The peak appeared when the initial electric field was applied along the $\mathrm{c}$ axis of the crystal and the peak shape resembles that of ferroelectric order-disorder transition. This observation supports that ice XI has a polar structure along the $\mathrm{c}$ axis.

Why does the proton ordering in ice $\mathrm{I}_{\mathrm{h}}$ not occur completely, as in many ordering transitions? As has been stated earlier, the entropy of transition depends on the amount of the dopant. This indicates that the dopant does not uniformly accelerate the orientational mobility of all the water dipoles but affect the water molecules surrounding the dopant to induce the ordering transition in micro-domains. Therefore, the low solubility of $\mathrm{KOH}$ in the lattice limits the crystal to realize the fully ordered ice. The second factor is the effect of annealing time. When an ice sample $\left(x=1.8 \times 10^{-3}\right)$ was annealed at $63 \sim 68 \mathrm{~K}$ for about one month, the entropy change increased to $2.81 \mathrm{~J} \mathrm{~K}^{-1} \mathrm{~mol}^{-1}$, corresponding to $82 \%$ ordering. One possible explanation for this finding is that the final stage of ordering is difficult to achieve kinetically. As the ordered domains grow, the water dipoles remaining in disordered state undergo frustration under the circumstances of surrounding microdomains with various polarizations. Thus the proton ordering process in ice $I_{h}$ is of extremely sluggish nature particularly at the final stage. On the other hand, Johari discussed the incomplete nature of the transformation by increasing strain energy at the twophase boundary in a polycrystalline solid. ${ }^{23)}$ He proposed that the magnitude of the strain energy did not vary with the amount of the low temperature phase but did so with the nature of the dopant.

In order to examine the relative importance of proton ordering on the heat transportation, the thermal conductivity of $\mathrm{KOH}$-doped ice was measured between 60 and $273 \mathrm{~K}$ as a function of pressure up to $1 \mathrm{GPa}^{24)}$ The measurement was done by using a transient hot-wire method with a Ni wire as a probe. The wire is placed horizontally in a ring of constant radius within a temperature-controlled Teflon cell that contains a $\mathrm{KOH}$ aqueous solution with $x_{\mathrm{KOH}}=1.8 \times 10^{-3}$. The sample was 


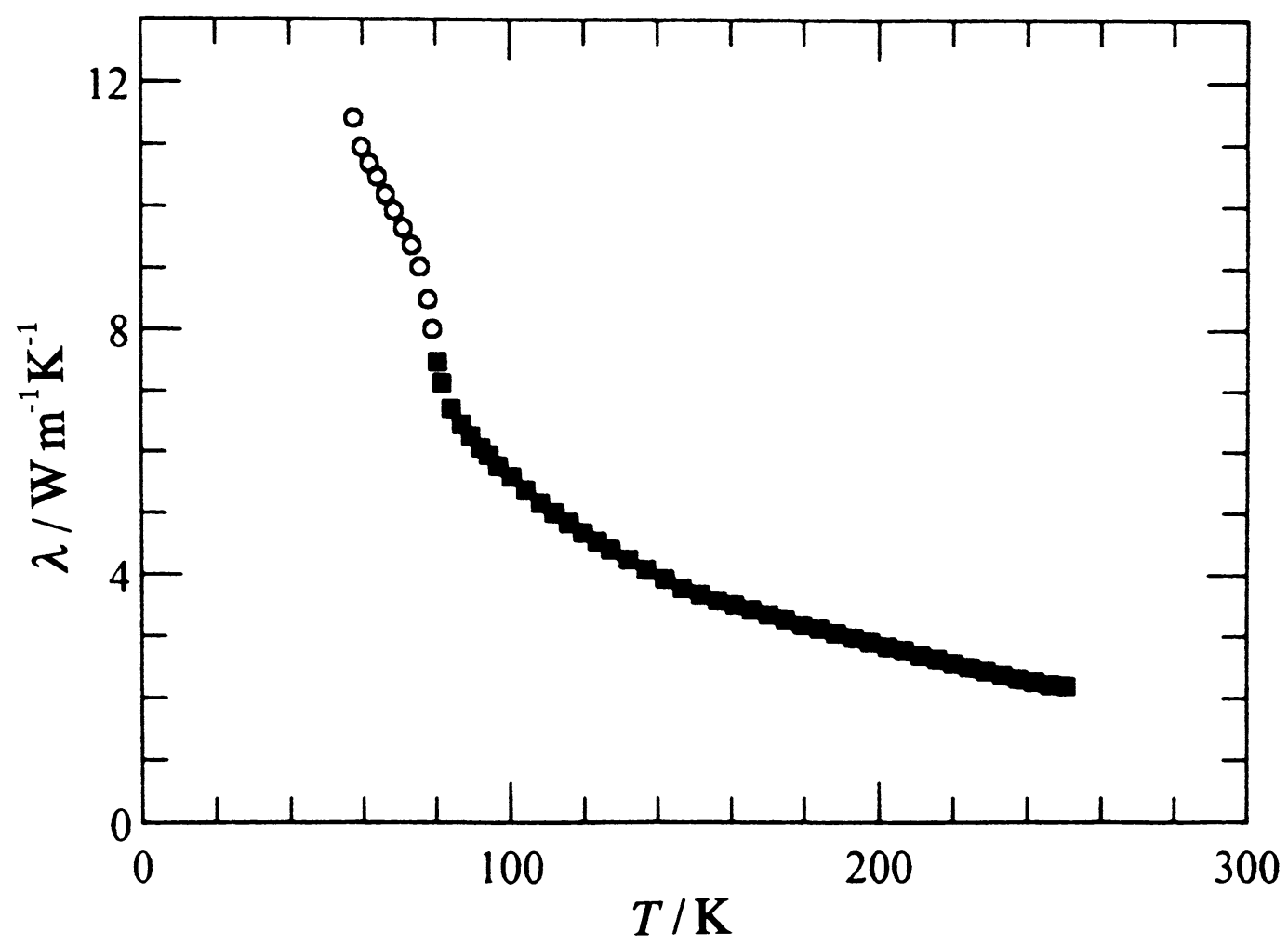

Fig. 6. Thermal conductivity $\lambda$ of $\mathrm{KOH}$-doped $\left(x_{\mathrm{KOH}}=1.8 \times 10^{-4}\right)$ ice.

annealed at $65 \mathrm{~K}$ for three days. The thermal conductivity $\lambda$ measured at constant pressure of $160 \mathrm{MPa}$ is plotted in Fig. 6 as a function of temperature. The temperature dependence of $\lambda$ for both phases $I_{h}$ and XI is characteristic of crystals for which the thermal conductivity is inversely proportional to temperature $T$, arising from "Umklapp" processes of phonons in the region above the Debye temperature. The effect of proton disordering in ice is reflected as a decrease in $\lambda$ by $\sim 17 \%$ at $72 \mathrm{~K}$. This decrease can be accounted for by an increase in lattice anharmonicity which might be caused by the orientational disordering.

From the pressure dependence of the thermal conductivity, measured at $65 \mathrm{~K}$, it is possible to derive the density dependence of $\lambda$ by using data on the isothermal compressibility. The quantity designated as the Bridgman parameter $g$ is defined by $g=$ $\left.(\partial \ln \lambda / \partial \ln \rho)_{T}\right]$, where $\rho$ is the density. Again the effect of proton disordering can be observed in the value of $g$. The value $g$ for phase XI is 0.2 , in contrast to -2.8 for $\mathrm{I}_{\mathrm{h}}$ determined at the same temperature of $65 \mathrm{~K}$. Crystalline substances exhibit generally $g$ values greater than 6 . Water possesses many exceptional physical and chemical properties even in its crystalline forms.

Cubic ice. Cubic ice $I_{c}$ exists also in nature and is a metastable modification of ice at atmospheric pressure. One evidence of the natural occurrence of ice $I_{c}$ is the Scheiner' halo ${ }^{25)}$ that appears rarely at the angle of $28^{\circ}$ around the sun or the moon. Ice $\mathrm{I}_{c}$ can be produced in laboratory by warming either amorphous ice or any high-pressure forms of ice after depressurization at low temperatures. The latter method is suitable to prepare $I_{c}$ sample in quantity large enough to be subjected to high accuracy measurement. The formation of $\mathrm{I}_{c}$ takes place at around $140 \mathrm{~K}$. The ice $\mathrm{I}_{\mathrm{c}}$ thus produced transforms slowly to $\mathrm{I}_{\mathrm{h}}$ over the temperature range $170 \sim 220 \mathrm{~K}$ with heat evolution of about $40 \mathrm{~J} \mathrm{~mol}^{-1}{ }^{26)}$ Owing to the metastability relative to $\mathrm{I}_{\mathrm{h}}$, ice $\mathrm{I}_{\mathrm{c}}$ cannot have citizenship in the phase diagram of ice. Ice $I_{c}$ is structurally similar to ice $I_{h}$, with an almost identical tetrahedral arrangement of the nearest neighbors. The spatial arrangement of the oxygen atoms is the same as that of diamond. Many experimental facts show that ice $\mathrm{I}_{\mathrm{c}}$ has proton-disordered structure. It is interesting therefore to examine 


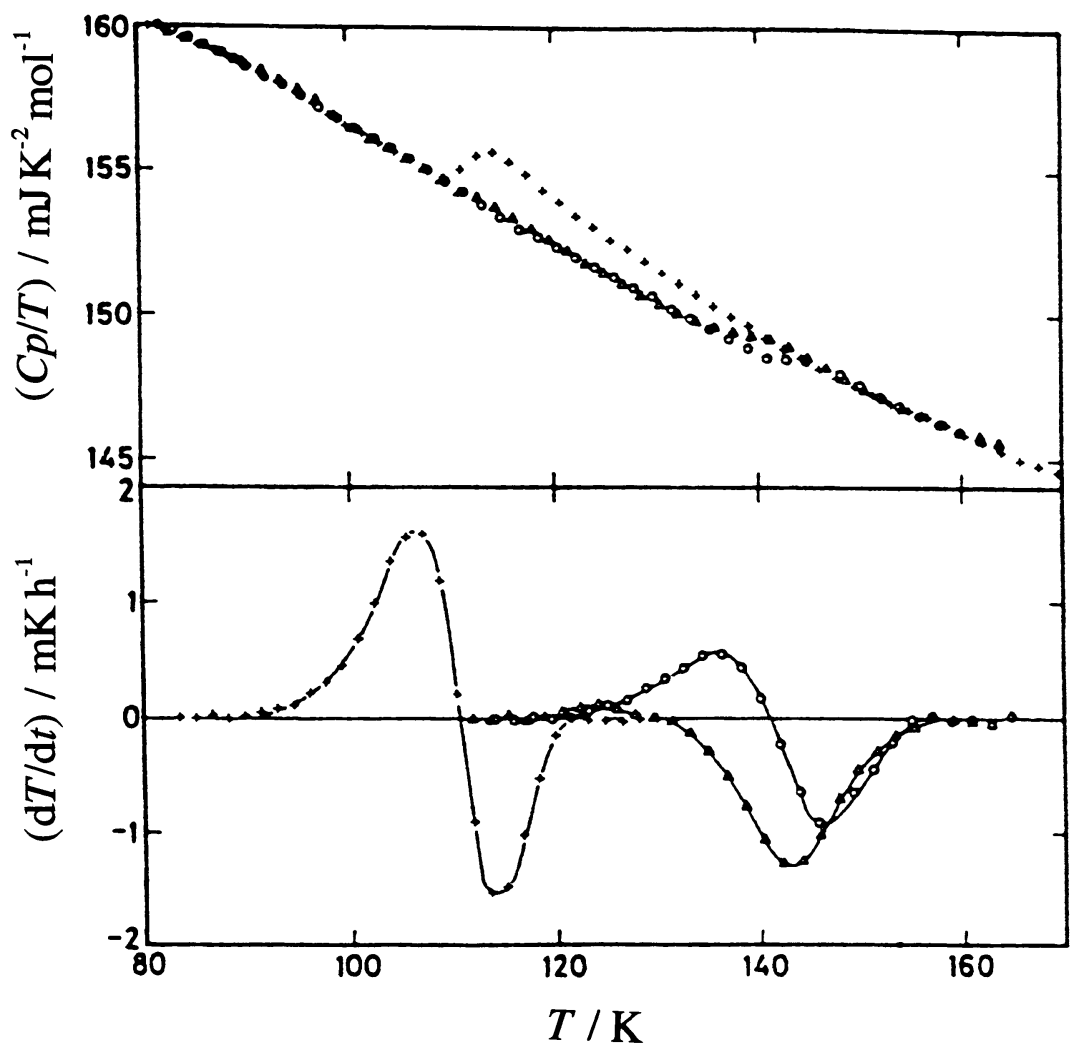

Fig. 7. (Top) Encraty $\left(C_{p} / T\right)$ and (bottom) spontaneous temperature drift rate of hexagonal and cubic ices plotted as a function of temperature. $\bigcirc ; \mathrm{I}_{c}$ cooled at $0.8 \mathrm{~K} \mathrm{~min}^{-1}$. $\mathbf{\Delta} ; \mathrm{I}_{c}$ annealed at $133 \mathrm{~K}$ for $21 \mathrm{~h} .+; \mathrm{I}_{\mathrm{h}}$ cooled at $0.6 \mathrm{~K} \mathrm{~min}^{-1}$.

whether the freezing out of the water reorientational motion can be found in $\mathrm{I}_{\mathrm{c}}$ and whether the dopant $\mathrm{KOH}$ works to release the frozen-in disordered state, as in the case of $I_{h}$.

A purified water sample was crystallized at $265 \mathrm{~K}$ and then pressurized by using He gas up to $250 \mathrm{MPa}$ to produce ice III in a high-pressure adiabatic calorimeter. ${ }^{27}$ ) The sample was cooled to $77 \mathrm{~K}$ and the pressure was released. Formation of cubic ice occurred at around 155 $\mathrm{K}$ on warming. Then the sample was cooled down to $13 \mathrm{~K}$ after annealing at $160 \mathrm{~K}$ and the calorimetric measurement was started. The heat capacity of $I_{c}$ was similar in magnitude to that of $\mathrm{I}_{\mathrm{h}}$ within $\pm 0.08 \mathrm{~J} \mathrm{~K}^{-1} \mathrm{~mol}^{-1}$ in the temperature range $13 \sim 100 \mathrm{~K}$. As expected, a freezing process was observed at around $143 \mathrm{~K}$ that is $33 \mathrm{~K}$ higher than that of $\mathrm{I}_{\mathrm{h}}$. A quantity called encraty $\left(C_{p} / T\right)$ and spontaneous temperature drift rate observed in each equilibration period are drawn in Fig. 7 as a function of temperature, respectively. Also plotted in the figure are the corresponding quantities of $\mathrm{I}_{\mathrm{h}}$ for a comparison. The encraty is convenient in the sense that it can magnify any small heat-capacity anomaly in the temperature range where the heat capacity changes rapidly with temperature. The same experiment was done for $\mathrm{KOH}$-doped ice $\mathrm{I}_{\mathrm{c}}$. The corresponding glass transition was found to be lower by $30 \mathrm{~K}$. No heat capacity anomaly ascribable to a transition into a hypothetical ordered phase was observed. This may arise from the fact that pressurization of ice induces rejection of ionic impurities from the specimen. ${ }^{28)}$ Even under such conditions, a residual portion escaped from the segregation acted to enhance the orientational mobility of the water molecules. The rate of spontaneous temperature change was analyzed to derive the enthalpy relaxation time governing the freezing process. The activation energy $38.0 \mathrm{~kJ} \mathrm{~mol}^{-1}$ for pure $I_{c}$ was found to be lowered to $14.9 \mathrm{~kJ} \mathrm{~mol}^{-1}$ for the doped sample. An aerosol method, in which $\mathrm{KOH}$ aqueous solution is atomized ultrasonically to form microdroplets and condensed on a cryoplate, will be an appropriate candidate for the preparation of doped $I_{c}$ 


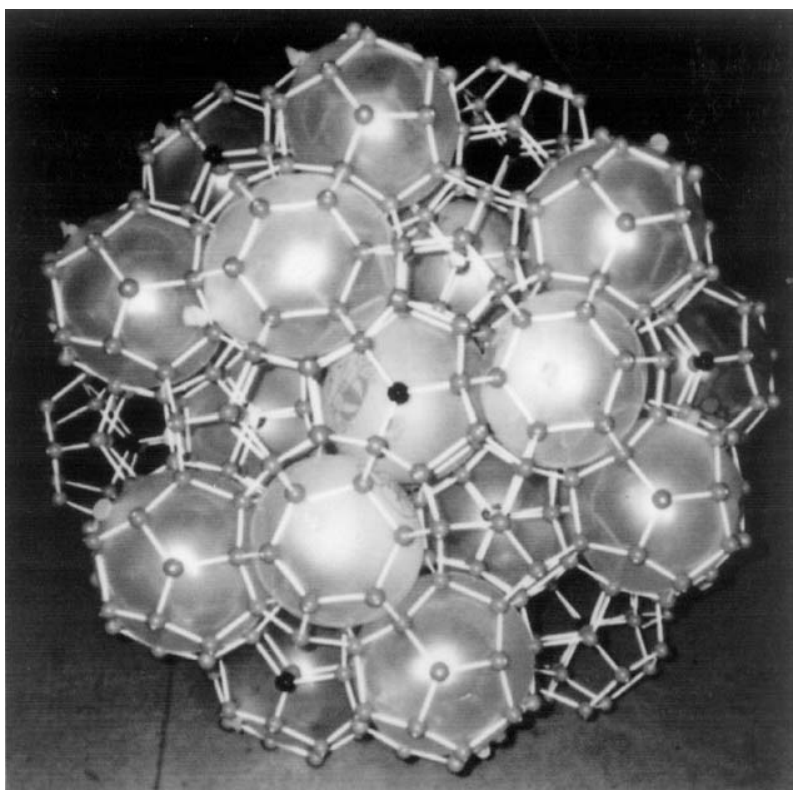

Fig. 8. Structure model of the type II clathrate hydrates.

sample. This has not yet been done owing to the experimental difficulty. The ordering transition of $I_{c}$ is expected theoretically to occur at $60 \mathrm{~K}^{29)}$

Clathrate hydrate. Hydrates of natural gas such as methane and ethane were found to exist in large quantity under the ocean bed or the permafrost region, and attracted much attention as a new energy resource replaceable to petroleum. In spite of the technological importance, only a few calorimetric measurements for the clathrate hydrates have been reported. The concept of clathrate compound was introduced for the structural combinations of two substances in which strong mutual bindings of molecules of one sort makes a firm enclosure of the other molecules. ${ }^{30)}$ In the clathrate hydrates, water molecules form three-dimensional hydrogen-bonded networks to make two types of cages which accommodate guest molecules of suitable size and shape without any specific or chemical interactions. There is virtually no chemical bonding between the host and guest molecules. The guests are, nevertheless, firmly constrained by high-energy barriers. Of particular concern in this article is the type II hydrate, in which the cubic unit cell is composed of 16 pentagonal dodecahedral and 8 hexakaidecahedral cages formed by 136 water molecules. Most of the guest molecules in the type II hydrates are enclathrated in the latter cages, so that the stoichiometric formula for the full occupation of a guest molecule $\mathrm{G}$ becomes $\mathrm{G} \cdot(136 / 8) \mathrm{H}_{2} \mathrm{O}$, or $\mathrm{G} \cdot 17 \mathrm{H}_{2} \mathrm{O}$. A structural model for the type II hydrates is shown in Fig. 8 , where the cages including balloon is the larger ones. One of the fascinating problems in the clathrate hydrates is that both the host and guest molecules are in disordered state at high temperatures. ${ }^{31)}$ For the clathrate hydrates encaging dipolar guest species, two kinds of dielectric relaxation can be generally observed. The one occurring at around $160 \mathrm{~K}$ for $1 \mathrm{kHz}$ is ascribed to freezing out of the water dipoles and resembles closely that of hexagonal ice $I_{h}$. The other occurring at cryogenic temperatures as low as $30 \mathrm{~K}$ is due to freezing out of the guest motion, indicating highly orientational mobility of the guest molecules inside the cavities of almost spherical symmetry. Most probably, the ultra-slow dynamics of the water molecules at low temperatures hinders the host lattice in realizing an ordered structure, as in the case of $I_{h}$.

In fact, acetone (Ac) clathrate hydrate $\mathrm{Ac} \cdot 17 \mathrm{H}_{2} \mathrm{O}$ exhibited a glass transition at about $90 \mathrm{~K}^{32)}$ Exothermic and endothermic processes associated with the glass transition have already been shown in Fig. 2 . The sample was cooled with a rate of about $2.4 \mathrm{~K}$ $\mathrm{min}^{-1}$, and the first series of measurements were carried out. Exothermic followed by endothermic processes characteristic of glass transition were observed. Then the sample was annealed at $80 \mathrm{~K}$ for $30 \mathrm{~h}$. Only endothermic processes appeared for the annealed sample. The value of $\beta$ determined by the calorimetric method was in the range $0.5 \sim 0.7$ over the temperature range $70 \sim 90 \mathrm{~K}$. An Arrhenius plot of the enthalpy relaxation time versus reciprocal temperature gave activation energy for the process to be $21.2 \mathrm{~kJ} \mathrm{~mol}^{-1}$, which was similar in magnitude to that of pure ice $\mathrm{I}_{\mathrm{h}}, 24 \mathrm{~kJ} \mathrm{~mol}^{-1}$. In order to accelerate the water motion, the same dopant $\mathrm{KOH}$ was incorporated into the hydrate crystal with the concentration of $x_{\mathrm{KOH}}=1.8 \times 10^{-4}$. As expected, the proton ordering occurred at $46.6 \mathrm{~K}$ with the associated entropy change of $2.4 \mathrm{~J} \mathrm{~K}^{-1}$ (mol of water) $)^{-1}$. The experimental heat capacities for pure and doped samples are drawn in Fig. 9, where the $C_{p}$ data are referred to one mole of water. The catalytic action of the same dopant was more dramatic, because the transformation proceeded much faster in the hydrate crystal than in ice $\mathrm{I}_{\mathrm{h}}$. A long tail of the excess heat capacity above $T_{\text {trs }}$ is noticeable. The dielectric relaxation of the guest Ac molecules was found to disappear below $T_{\text {trs }}$. No anomalous behavior was observed at around $30 \mathrm{~K}$, where the second dielectric relaxation due to the guest dipoles was found in the pure sample. Most probably the ordering of the 


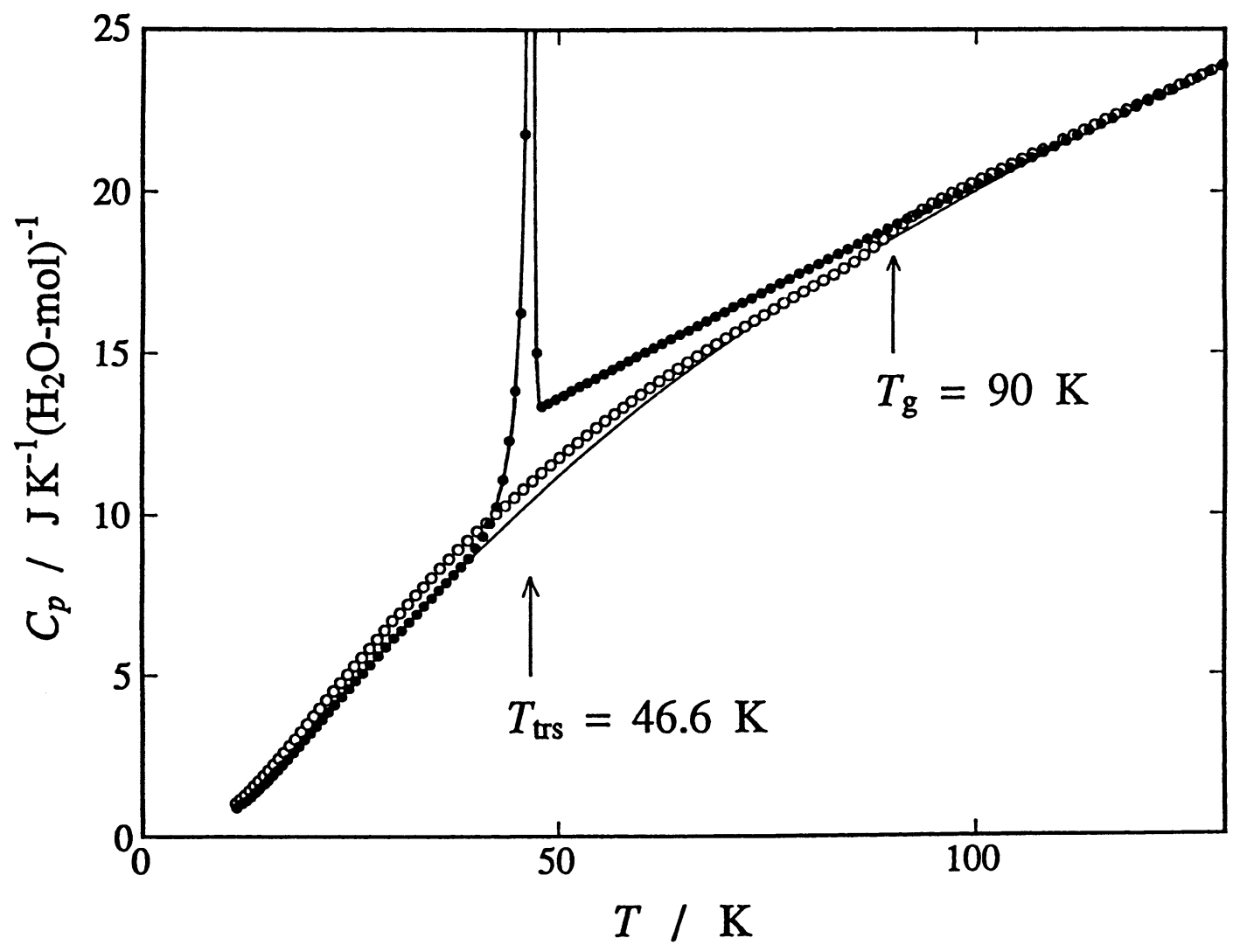

Fig. 9. Heat capacities of pure $(\bigcirc)$ and $\mathrm{KOH}$-doped $\left(x_{\mathrm{KOH}}=1.8 \times 10^{-4}\right)(O)$ acetone hydrates.

host dipoles produced a strong electric field at the guest site and this local field forced the guest dipoles to align along a preferred orientation inside the cage.

Essentially the same phenomena were found for the type II hydrates enclathrating tetrahydrofuran (THF) and trimethylene oxide (TMO). The transition temperature was $61.9 \mathrm{~K}$ for the former and $34.5 \mathrm{~K}$ for the latter, respectively. Excess heat capacity and the corresponding entropy associated with the phase transitions for the three clathrate hydrates are summarized in Fig. 10. Some physical properties of the guest molecules are affecting the proton-ordering temperature of the host lattice. Dipole moment and van der Waals radius would be important factors to be taken into account. But it is not clear at the moment which property of the guest molecule is predominantly effective in determining the transition temperature. In each case, the excess entropy associated with the transition is composed of two contributions; a discontinuous part at the transition temperature $T_{\text {trs }}$ and a continuously increasing part extending far above $T_{\text {trs }}$ up to $110 \mathrm{~K}$. The smaller the discontinuous jump, the larger the high-temperature tail. The high-temperature asymptotic value of the entropy of transition is approximately $2.4 \mathrm{~J} \mathrm{~K}^{-1}$ (mol of water) $)^{-1}$ for the three hydrates, and is close to ice $\mathrm{I}_{\mathrm{h}}$ doped with $x=$ $1.8 \times 10^{-3} \mathrm{KOH}$. The dielectric measurements of the three hydrate crystals for pure and doped samples have clarified the dynamics of the host and guest molecules. ${ }^{33}$ Calorimetric and dielectric relaxation times determined for pure and doped ice, as well as for the clathrate hydrates are summarized in Fig. 11. The dielectric data for pure and $\mathrm{KOH}$-doped ices were taken from a literature ${ }^{34)}$ For the doped Ac hydrate crystal, the dielectric relaxation time for water dipoles was shortened by a factor of $10^{-10}$ at about $70 \mathrm{~K}$. In addition, the activation energy decreased to $7.4 \mathrm{~kJ} \mathrm{~mol}^{-1}$ for THF, $8.5 \mathrm{~kJ} \mathrm{~mol}^{-1}$ for Ac, and $9.0 \mathrm{~kJ} \mathrm{~mol}^{-1}$ for TMO, respectively. Thus the dopant even at minute amounts dramatically shortened the relaxation time and diminished the activation energy for the water reorientational process in the lattice of the hydrate crystals.

Thermal conductivities of $\mathrm{KOH}\left(x=1.8 \times 10^{-4}\right)$ - 

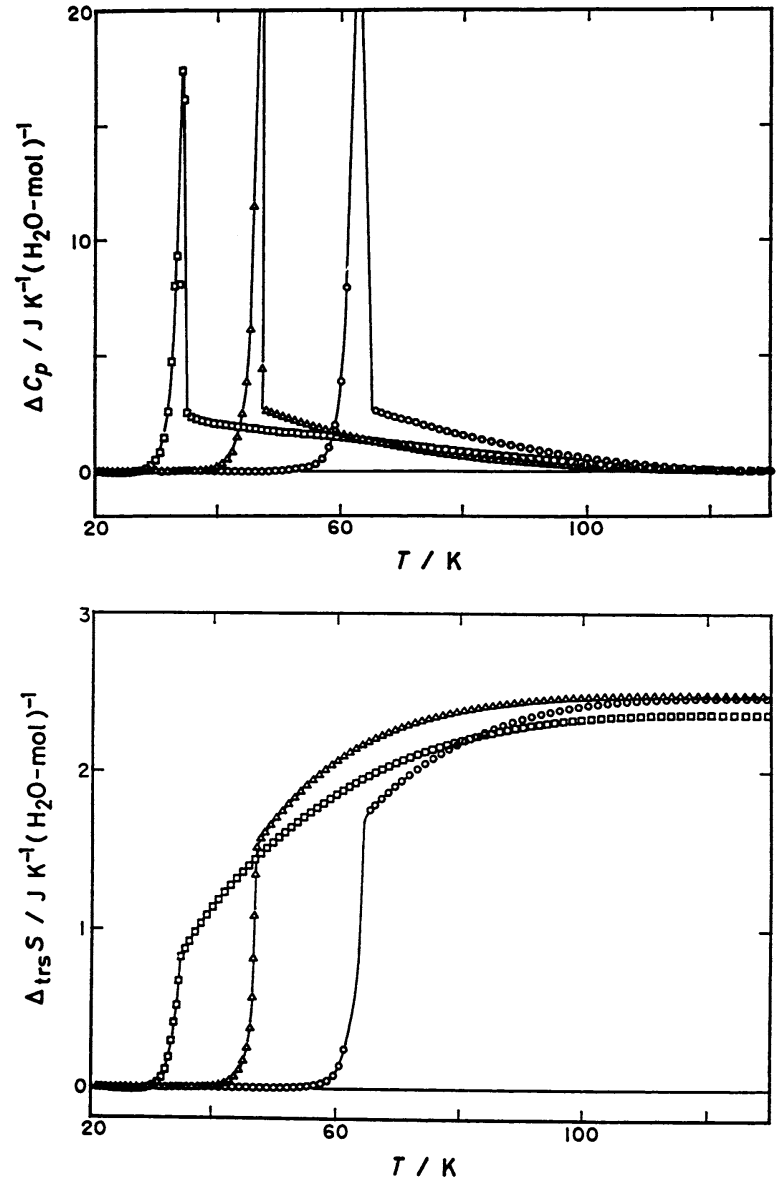

Fig.10. Excess heat capacity (top) and entropy (bottom) associated with ordering transitions of three clathrate hydrates encaging trimethylene oxide (TMO, left), acetone (Ac, center), and tetrahydrofuran (THF, right), respectively.

doped normal and KOD $\left(x=1.8 \times 10^{-4}\right)$-doped deuterated THF clathrate hydrates were measured in the temperature range 55 250 $\mathrm{K}$ and at pressures up to 0.16 GPa by the same method as ice $\mathrm{I}_{\mathrm{h}}{ }^{35)}$ At isobaric heating, the thermal conductivity for both samples exhibited discontinuous drop by $15 \%$ at the respective transitions. The amount of decrease in $\kappa$ just corresponds to that in $I_{h}$ in association with the proton disordering. The value of $\kappa$ for deuterated sample is higher by $5 \%$ than that of the normal sample. Both the values for the low-temperature (LT) and high-temperature (HT) phases increase slightly with increasing temperature and, hence, these crystalline phases exhibited both a glass-like behavior in the heat transport process. This unexpected behavior has already been reported for pure THF clathrate hydrate ${ }^{36)}$ Since it is unusual for crystals to exhibit glass- like $\kappa$, a reasonable conclusion is that the same mechanism causes the glassy behavior in both of the LT and HT phases. In the case that the mechanism is strong phonon scattering, it should be almost equally strong in both phases. This consideration rules out immediately molecular orientational disorder as the main scattering source, since the calorimetric and dielectric measurements show that the LT phase is ordered while the HT phase is disordered ${ }^{36), 37)}$ Our results support the resonance scattering model ${ }^{38)}$ which states that the acoustic phonons interact with the guest vibrations to yield phonon scattering. In fact, our $\kappa$ data were reproduced fairly well by this model with appropriate parameters.

It is worthy mentioning the important role played by the dopant. As is well known, scientific knowledge is generally based on our limited observations of experiments that are undertaken on the laboratory time-scale. Practically all the chemical equilibria are pseudo-equilibria in the sense that they are not related to the absolute minimum of the Gibbs energy with respect to all virtual processes, but only with respect to the processes that could take place within a reasonable time. Phase transitions in disordered crystals cannot escape from this situation. As clarified by the third law of thermodynamics, all crystalline substances prefer ordered states as the temperature approaches zero kelvin. This statement is correct only when all the aspects of a system are in internal equilibrium. The "internal equilibrium" can be established in most cases within a reasonable time. Thus many phase transitions due to ordering of some aspects of a system have been observed with whole shape of the associated heat capacity anomaly. On the other hand, each degree of freedom has a characteristic relaxation time that changes more or less with temperature. If the relaxation time becomes too long to establish the equilibrium, the associated physical quantities cannot follow the equilibrium values on cooling below a certain temperature, resulting in a frozen-in disordered nonequilibrium state. A particular kind of impurity doped into the lattice acted on the non-equilibrium system as catalysis for releasing the immobilized state and recovering the thermal equilibrium within our observation time. Thus, the phase transformation and glass transition are the phenomena encountered during continuous cooling of any disordered systems, depending on the dynamical situation of the constitute molecules. In relation to this fact, one may raise a question "What kind of physical or chemical defects will release the immobilized state of liquid?" If a particular dopant were discovered 


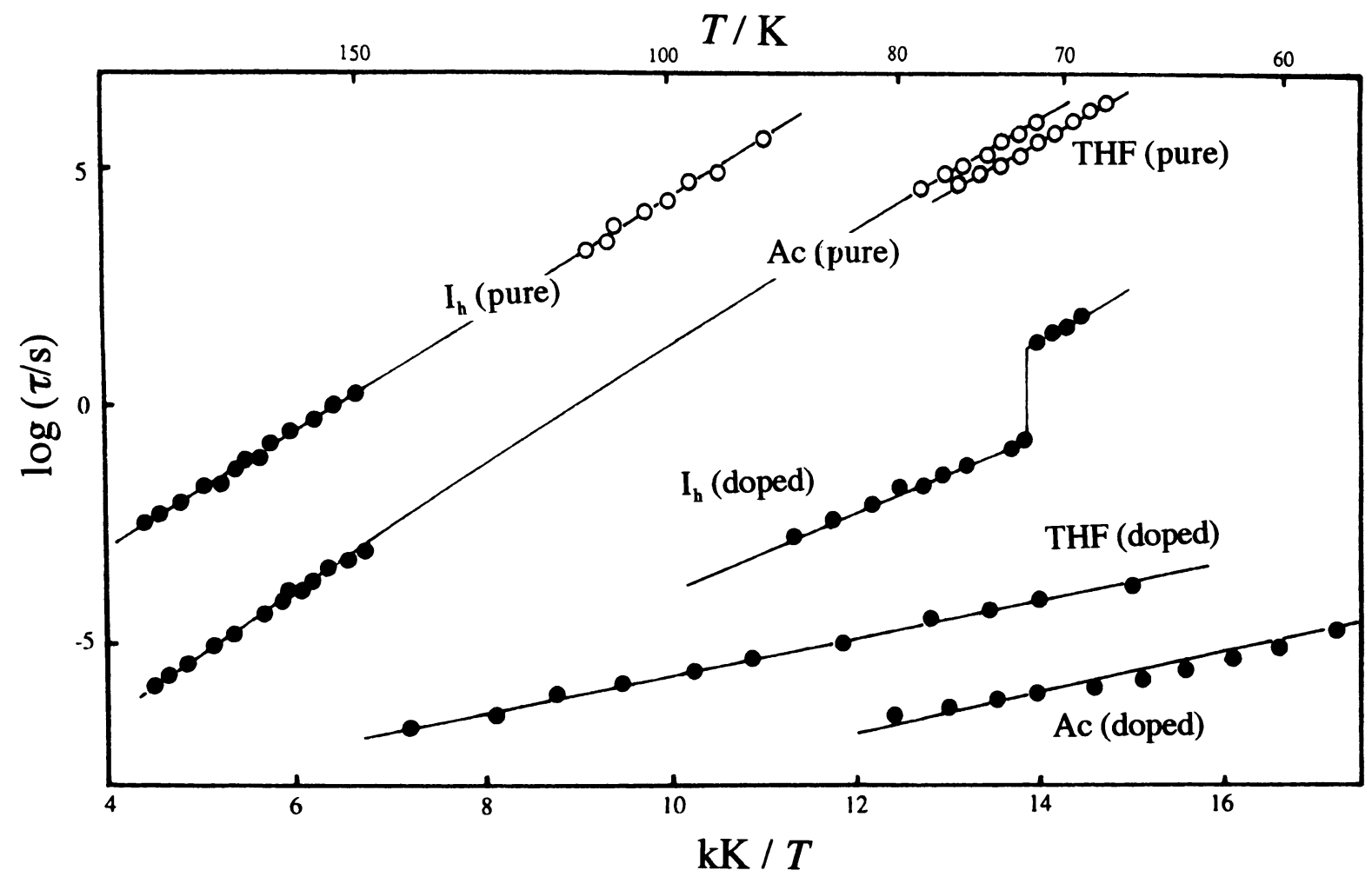

Fig.11. Arrhenius plots of the relaxation times for ice and clathrate hydrates determined by calorimetric and dielectric measurements.

luckily for this purpose, we will be blessed to observe in reality what will happen at the Kauzmann temperature of a liquid. As a matter of fact, it is not easy for us to imagine some disordered solids possessing the same amount of entropy of the crystal. The ground state of liquid without configurational entropy is designated as an "ideal glass".

Acknowledgments. The author would like to express his sincere thanks to the late Professor Isamu Nitta and Professor Emeritus Syûzô Seki, M. J. A., who introduced him to these intriguing fields. Thanks are extended to many former co-workers including graduate and undergraduate students in Osaka University. Without their enthusiastic collaboration, the present article could not have been possible. These works were financially supported mainly by Ministry of Education, Culture, Sports, Science and Technology and partly by the British Council as well as the Swedish Natural Science Council.

\section{References}

1) Brown, S. (1985) Relaxation in Viscous Liquids and Glasses. Am. Ceram. Soc., Columbus.

2) (a) Suga, H., and Seki, S. (1974) J. Non-cryst. Solids 16, 171-194; (b) Suga, H., and Seki, S. (1980) Faraday Discussion 69, 221-240; (c) Suga, H. (1993) J. Chem. Thermodyn. 25, 463-484

3) Suga, H., and Seki, S. (1988) Encyclopedia of Materials Sciences and Engineering Suppl. I (ed. Cahn, W.). Pergamon Press, London, pp. 190-192.

4) Parsonage, N. G., and Staveley, L. A. K. (1978) Disorder in Crystals. Clarendon Press, Oxford.

5) Eisenberg, D., and Kauzmann, W. (1969) The Structure and Properties of Water. Clarendon Press, Oxford.

6) (a) Giauque, W. F., and Stout, J. W. (1936) J. Am. Chem. Soc. 58, 1144-1150; (b) Pauling, L. (1935) J. Am. Chem. Soc. 57, 2680-2684; (c) Bernal, J. D., and Fowler, R. H. (1933) J. Chem. Phys. 1, 515-548.

7) Suga, H., and Matsuo, T. (1989) Pure Appl. Chem. 61, 1123-1132.

8) (a) Suga, H. (1986) Ann. New York Acad. Sci. 484, 248-263;

(b) Suga, H. (1997) Thermochim. Acta 300, 117-126.

9) Ohta, T., Yamamuro, O., and Suga, H. (1994) J. Chem. 
Thermodyn. 26, 319-331.

10) Kishimoto, I., Pinvidic, J. J., Matsuo, T., and Suga, H. (1991) Proc. Jpn. Acad., Ser. B 67, 66-71.

11) Haida, O., Matsuo, T., Suga, H., and Seki, S. (1974) J. Chem. Thermodyn. 6, 815-825.

12) Pitzer, K. S., and Polissar, J. (1956) J. Phys. Chem. 60, 1140-1142.

13) (a) Ida, M., Nakatani, N., Imai, K., and Kawada, S. (1966) Sci. Rep. Kanazawa Univ. 11, 13-22; (b) Kawada, S. (1972) J. Phys. Soc. Jpn. 32, 1442.

14) (a)Tajima, Y., Matsuo, T., and Suga, H. (1982) Nature 299, 810-812; (b) Tajima, Y., Matsuo, T., and Suga, H. (1984) J. Phys. Chem. Solids 45, 1135-1144; (c) Matsuo, T., Tajima, Y., and Suga, H. (1986) J. Phys. Chem. Solids 47, 165-173.

15) Yamamuro, O., Oguni, M., Matsuo, T., and Suga, H. (1987) J. Chem. Phys. 86, 5137-5140.

16) Leadbetter, A. J., Ward, R. C., Clark, J. W., Tucker, P. A., Matsuo, T., and Suga, H. (1985) J. Chem. Phys. 82, 424-428.

17) Suga, H., Matsuo, T., and Yamamuro, O. (1992) Pure Appl. Chem. 64, 17-26.

18) (a) Howe, R., and Whitworth, R. W. (1989) J. Chem. Phys. 90, 4450-4453; (b) Line, C. M. B., and Whitworth, R. W. (1996) J. Chem. Phys. 104, 10008-10013.

19) Howe, R. (1987) J. de Phys. 48, 599-608.

20) Davidson, E. R., and Morokuma, K. (1984) J. Chem. Phys. 81, 3741-3742.

21) Pisani, C., Casassa, S., and Ugliengo, P. (1996) Chem. Phys. Lett. 253, 201-208.

22) Jackson, S. M., and Whitworth, R. W. (1995) J. Chem. Phys. 103, 7647-7648.

23) Johari, J. P. (1999) Phil. Mag. A79, 1479-1492.

24) Andersson, O., and Suga, H. (1994) Phys. Rev. B50, 6583-6588.

25) Whalley, E. (1983) J. Phys. Chem. 87, 4174-4179.
26) Sugisaki, M., Suga, H., and Seki, S. (1968) Bull. Chem. Soc. Jpn. 41, 2591-2599.

27) Yamamuro, O., Oguni, M., Matsuo, T., and Suga, H. (1987) J. Phys. Chem. Solids 48, 935-942.

28) Gough, S. R., and Davidson, D. W. (1970) J. Chem. Phys. 52 5442-5449.

29) Minagawa, I. (1983) J. Phys. Soc. Jpn. 52, 1641-1645.

30) Mak, T. C. W., and McMullan, R. K. (1965) J. Chem. Phys. 42, 2732-2737.

31) Davidson, D. W. (1988) Water - A Comprehensive Treatise (ed. Franks, F.). Vol. 2. Plenum Press, New York.

32) (a) Yamamuro, O., Oguni, M., Matsuo, T., and Suga, H. (1988) J. Phys. Chem. Solids 49, 425-434; (b) Yamamuro, O., and Suga, H. (1989) J. Thermal Anal. 35, 2025-2064; (c) Kuratomi, N., Yamamuro, O., Matsuo, T., and Suga, H. (1991) J. Chem. Thermodyn. 23, 485-494.

33) Suga, H., Matsuo, T., and Yamamuro, O. (1993) Supramolecular Chem. 1, 221-235.

34) Kawada, S., and Dohata, H. (1985) J. Phys. Soc. Jpn. 54, 477-479.

35) Andersson, O., and Suga, H. (1996) J. Phys. Chem. Solids 57, 125-132.

36) Ross, R. G., Andersson, P., and Bäckström, G. (1981) Nature 290, 322-323.

37) Handa, Y. P., and Cook, J. G. (1987) J. Phys. Chem. 91, 6327-6328.

38) (a) Tse, J. S., and White, M. A. (1988) J. Phys. Chem. 92 5006-5011; (b) Tse, J. S. (1994) J. Inclusion Phenomena 17, 259-266.

(Received Aug. 29, 2005; accepted Nov. 14, 2005)

\section{Profile}

Hiroshi Suga was born in 1930 and started his research career in 1953 with calorimetric studies of phase transitions in crystals, after graduating from the Faculty of Science at Osaka University. His research subject shifted gradually to non-equilibrium phenomena in molecular solids. He and his group have found that the glass transition was not a characteristic property of liquids but of wide occurrence in solids irrespective of the periodicity in their molecular arrangements. He became Professor at Osaka University in 1979, and Kinki University in 1993. His research object covers a wide range of substance including minerals, inorganic as well as organic compounds, synthetic and biological polymers, being a consequence of general validity of the underlying thermodynamic laws. His international reputation earned honors, such as the Huffman Award from the US Calorimetry Conference (1992), the Kurnakov Medal from Russia (1994), the Swietoslawski Medal from Poland (1997), and the ICTAC-SETARAM Award (2004). He was President of the Japan Society of Calorimetry and Thermal Analysis during 1991-1992.

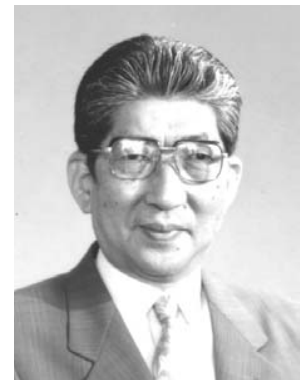

